\title{
Characterization and stability of nanostructured lipid carriers as drug delivery system.
}

\begin{abstract}
Recently more focus has been put to the development of innovative drug-delivery systems that includes polymer nanoparticles, emulsions and liposomes and solid lipid nanoparticles (SLNs). The SLNs have been proposed to be an alternative colloidal drug delivery system. The aim of this study was preparation and characterization of solid lipid nanoparticle (SLN) using varieties of emulsifier for encapsulation of the drug with poor water solubility. In these study four types of solid lipid nanoparticles were prepared based on different compositions of palm oil (S154) and lecithin (Lipoid 100) using the high pressure homogenization method. The SLN formulation had the following (palm oil+lecithin) compositions: SLN-01 $(90+10 \%$, respectively), SLN-02 (80+20\%, respectively), SLN-03 (70+30\%, respectively) and SLN-04 $(60+40 \%$, respectively). The SLNs were characterized and the optimum stability factors for one year storage determined. The parameters used to characterize the SLNs were particle size and polydispersity index (particle sizer), zeta potential (zetasizer), crystallinity (differential scanning calorimetry and wide angle X-ray diffraction), ultrastructure (transmission electron microscopy). Varying the palm oil and lecithin compositions resulted in SLNs of variable sizes and zeta potentials. The particle sizes of SLN-01, SLN-02, SLN-03 and SLN-04 were $298.40 \pm 11.80,255.40 \pm 3.20,145.00 \pm 3.39$ and $273.00 \pm 86.50 \mathrm{~nm}$, respectively, while the zeta potentials were $-19.44 \pm 60.00,-19.50 \pm 1.80,-17.83 \pm 10.00$ and $-13.33 \pm 2.30 \mathrm{mV}$, respectively. Thermoanalysis and X-ray diffraction analysis showed that the SLNs had lower crystallinity than bulk lipid. The SLNs were generally round and uniform in shape under transmission electron microscopy. The SLN dimensional data suggested they had high quality physicochemical characteristics, which are conducive for the loading of poor water solubility drugs.
\end{abstract}

Keyword: Characterizations; Colloidal system; Drug delivery; Solid lipid nanoparticles. 